\title{
Analysis of Vibrations Generated by the Presence of Corrugation in a Modeled Tram Track
}

\author{
Julia I. Real Herráiz, ${ }^{1}$ Silvia Morales-Ivorra, ${ }^{1}$ Clara Zamorano Martín, ${ }^{2}$ \\ and Vicente Soler Basauri ${ }^{3}$ \\ ${ }^{1}$ University Institute for Multidisciplinary Mathematics, Polytechnic University of Valencia, 46022 Valencia, Spain \\ ${ }^{2}$ Foundation for the Research and Engineering in Railways, 160 Serrano, 28002 Madrid, Spain \\ ${ }^{3}$ Department of Applied Mathematics, Polytechnic University of Valencia, 46022 Valencia, Spain
}

Correspondence should be addressed to Julia I. Real Herráiz; jureaher@tra.upv.es

Received 7 August 2014; Revised 2 January 2015; Accepted 20 January 2015

Academic Editor: Xiaojun Wang

Copyright (C) 2015 Julia I. Real Herráiz et al. This is an open access article distributed under the Creative Commons Attribution License, which permits unrestricted use, distribution, and reproduction in any medium, provided the original work is properly cited.

In recent years, there has been a significant increase in the development of the railway system. Despite the huge benefits of railways, one of the main drawbacks of this mode of transport is vibrations caused by vehicles in service, especially in the case of trams circulating in urban areas. Moreover, this undesirable phenomenon may be exacerbated by the presence of irregularities in the railwheel contact. Thus, an analytical model able to reproduce the vibrational behavior of a real stretch of tram track was implemented. Besides, a simulation of different types of corrugation was carried out by calculating in an auxiliary model the dynamic overloads generated by corrugation. These dynamic overloads fed the main model to obtain the vibrations generated and then transmitted to the track.

\section{Introduction}

The development of new railway networks as well as the improvement of the already existing tracks has become a worldwide priority. At the same time, externalities derived from railway operations have been identified as a major drawback for this mode of transport, with vibrations being one of the externalities which must be highlighted. Vibrations, caused by vehicles in service, are propagated from the railway superstructure, and they may affect people or structures in the surrounding areas. Thus, major efforts have been focused in minimizing the potential effect of vibratory waves on elements placed outside of the transport system, being necessary to study their nature and mechanisms of generation, transmission, and propagation.

Railway vibrations are originated by the rail-wheel contact, so contact surfaces of both elements have a huge influence on the wave features of generated vibration, as stated by [1]. Tough different rail irregularities are able to modify the running surface and, therefore, cause specific vibrations; this study has been focused on the vibrational effects caused by corrugation.
Corrugation is a widespread pathology which can be found in all types of railroads. Reference [2] established that given a particular rail profile, corrugation can be considered, in one hand, both as a wavelength-fixing mechanism which depends on the dynamic features of the railroad and as a damage mechanism induced by dynamic loads. This finally results in a change in the rail profile determining new wavelengths as far as new damage, thus constituting a cyclic and progressive process. Besides, these authors classified the structural pathologies depending on the wavelength-fixing mechanism and on the damage mechanisms and proposed some treatments to reduce them. Reference [3] extended the research of causes of corrugation and suggested new treatments to prevent its progress.

More recently, [4, 5] after studying the development of corrugation by finite element models (FEM) in different types of railroads, we concluded that curves with small radii favor the appearance of wear, with the damage placed under those wheels circulating in the inner side of the curve.

Furthermore, at high speeds, the performance against corrugation is better in slab tracks than in ballasted tracks. A different point of view was contributed by [6], who analyzed 
the influence of pad stiffness when corrugation appeared in the low rail of a curve in Bilbao's underground. Those authors considered a multibody model for the vehicle and, applying [7] proposals, predicted frequencies at which corrugation is more likely to occur. Besides, they demonstrated that elastic pads reduce corrugation by suppressing some wavelengths that otherwise will be developed.

Different prediction models for corrugation have been described until now, acquiring special relevance to know how imperfections in rails surface affect the vibrations induced to the track. Dynamic overloads originated by corrugation were calculated by [8] and then implemented in a discrete FEM to assess the vibrations derived from it. They concluded that frequencies associated with those continuous defects can be determined by the quotient between train speed and the wavelength associated with this imperfection.

By using a three-dimensional model of wheel-rail interaction, [9] predicted the evolution of wear as well as its effect on the dynamic behavior of the track. According to this study, being the speed of the vehicle and the wavelength associated with corrugation constants, the larger the amplitude, the higher the dynamic overloads and wear derived from corrugation. Moreover, with the rest of parameters being constant, the greater the corrugation wavelength, the lower dynamic overloads and wear. In addition, the vertical stiffness of the different elements of the track was noted as a determining factor on the corrugation properties (i.e., amplitude and wavelength).

Reference [10], by using a FEM in a moving reference system, analyzed the vehicle-track interaction. On the surface of a rail, a sinusoidal corrugation was simulated, concluding that all the dynamic responses (displacement, loads, and acceleration) induced by this irregularity keep this sinusoidal shape. It was also found that the greater the severity of the defect, the higher the dynamic overloads generated. As for the speed, its influence on the dynamic response of the track was demonstrated. When forces in the wheel-rail contact were measured, it was found that both displacements and accelerations grew with speed.

In the present paper, an analytical model based on that used by [11] was implemented to reproduce the vibratory behavior of a tram track. Vibration data obtained in a campaign of measurements were used to calibrate the model.

Once proved the ability of the model to reproduce the vibratory phenomenon from the real track, corrugation will be introduced in the model and its effect on the vibrations transmitted to the ground will be analyzed. This will require the use of an auxiliary model, as that presented by [12] is able to calculate those dynamic overloads generated by corrugation and transmitted to the track. Thus, once the dynamic overloads are obtained, they will be introduced in the main model to calculate the vibrations generated by this irregularity. Furthermore, a sensibility analysis of the different parameters involved in the generation of dynamic overloads will be carried out to unravel those who are relevant in the vibration phenomenon and thus act on them.

This model may be useful to predict vibrations generated by the track corrugation and after establishing acceptable vibratory levels, it may allow determining the optimum

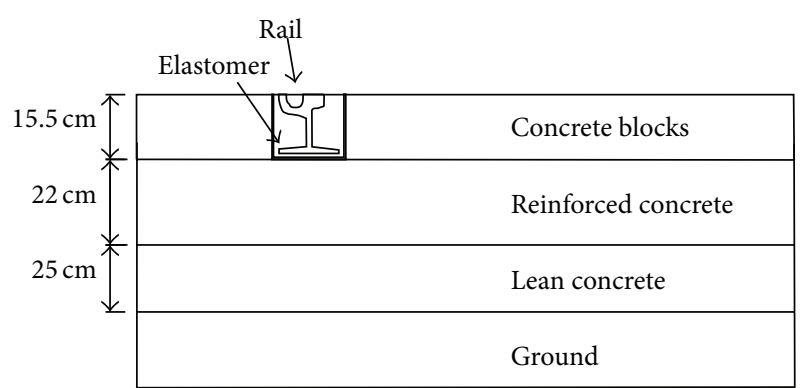

FIGURE 1

moment to carry out the maintenance operations. Furthermore, the present analytical model can also be employed to calculate those track overloads due to corrugation which generate not only an aggravation of the rail irregularities but also premature deterioration of the rest of the track, as stated by [3].

\section{Analytical Modelization of the Track}

The aim of this section is to describe the modelization process of the stretch of the tramway track studied, as well as its analytical resolution. In addition, the data, obtained in a measurement campaign and used to calibrate the model, will be presented. Thus, a model able to reproduce accurately the vibratory behavior of the studied track has been obtained and then discussed.

2.1. Description of the Tramway Track Stretch. The crosssection of the track studied is constituted by a Ph37N rail embedded in elastomeric material, which are housed in a $15.5 \mathrm{~cm}$ thick surface layer made of concrete blocks. The surface layer is on a reinforced concrete slab of $22 \mathrm{~cm}$ thickness, which rests on a $25 \mathrm{~cm}$ thick layer of lean concrete as shown in Figure 1.

The tram in service on this stretch of track is a Vossloh 4100 series as shown in Figure 2. Its service speed is $35 \mathrm{~km} / \mathrm{h}$-with $100 \mathrm{~km} / \mathrm{h}$ being its maximum speed-and transmits an axle load of $100 \mathrm{kN}$. The vehicle is composed of two motorized passenger cars at both extremes and another placed in the middle of them. The distance between axles of a same bogie is $2 \mathrm{~m}$, and the distances between bogies are in turn $12.54 \mathrm{~m}, 4.70 \mathrm{~m}$, and $12.54 \mathrm{~m}$.

2.2. Description of the Track Model. A bidimensional model able to calculate stresses and displacements is considered following [11, 13].

A system integrated by three layers representing the different materials that compose the track is developed. As stated in the previous section, a Phoenix rail and the elastomeric material are placed in the first layer and composed of concrete blocks. The second layer represents the concrete slab and the third one the lean concrete. Note that an indefinite depth for ground located under the last of these three layers (Boussinesq half space) is assumed. 


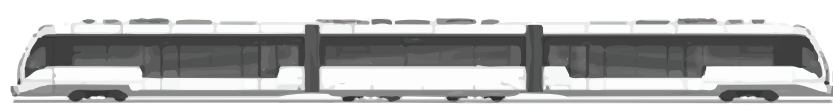

Figure 2

The rail has been represented as a Timoshenko beam following [14], with its movement equations being those described by [3]

$$
\begin{gathered}
\rho A \frac{\partial^{2} w}{\partial t^{2}}=\frac{\partial}{\partial x}\left(A_{k} G\left(\frac{\partial w}{\partial x}-\theta\right)\right)+q(x, t), \\
\rho I \frac{\partial^{2} \theta}{\partial t^{2}}=\frac{\partial}{\partial x}\left(E I \frac{\partial \theta}{\partial x}\right)+A_{k} G\left(\frac{\partial w}{\partial x}-\theta\right),
\end{gathered}
$$

where $A$ is the cross-sectional area, $A_{k}$ is the shear crosssectional area, $E$ corresponds to Young modulus, $I$ is the inertia in $y$-axis direction, $\rho$ is the rail mass density, $w$ is the vertical rail displacement, $\theta$ is the angular displacement, and $q(x, t)$ is the applied load depending on position and time.

Otherwise, the features of materials composing the layers are shown in Table 1.

By assuming a low viscosity between layers and following the research made by [15] the displacements can be expressed by means of the following vectorial equation:

$$
(\widehat{\lambda}+\widehat{\mu}) \nabla_{x, z}\left(\nabla_{x, z} \mathbf{d}\right)+\widehat{\mu} \nabla_{x, z}^{2} \mathbf{d}=\rho \frac{\partial^{2} \mathbf{d}}{\partial t^{2}},
$$

while $\mathbf{d}=(u(x, z, t), 0, v(x, z, t))$ represents the displacement of each layer, with $\rho$ being the mass density for each layer. Meanwhile, $\hat{\lambda}$ and $\hat{\mu}$ are the parameters employed to describe the viscoelastic behavior of each layer, as stated by [15]. Those parameters will be calibrated by using experimental data.

2.3. Load Modeling. When modeling loads, two different situations must be distinguished. On one hand, the quasistatic loads, which are due to the weight of the train traveling at a speed $V$-, must be taken into account. On the other hand, the dynamic overloads, which are the result of irregularities in the rail-wheel contact, will be discussed. These irregularities may be caused by defects in rails or wheels, joints between rails, or corrugation.

The introduction of the harmonic loads which represent the dynamic overloads in the model is made following [16]. These harmonic loads can be written as $P_{j}(t)=P_{0 j} \cos \left(\varrho_{j} t\right)$, where $P_{0 j}$ and $\omega_{j}$ represent, respectively, the magnitude and the characteristic angular frequency for each load.

The effect of quasistatic loads can be introduced as a dynamic overload with a null characteristic angular frequency, as stated by [13] or following the Zimmermann formulation, as described by [17]. Because of computational time reasons, in this paper the second procedure will be followed.
TABLE 1

\begin{tabular}{lccc}
\hline & $E(\mathrm{~Pa})$ & $\nu$ & $\rho\left(\mathrm{kg} / \mathrm{m}^{3}\right)$ \\
\hline Paving blocks & $2.25 * 10^{\wedge} 9$ & 0.25 & 2400 \\
Reinforced concrete & $2.73 * 10^{\wedge} 10$ & 0.25 & 2400 \\
Lean concrete & $2.25 * 10^{\wedge} 10$ & 0.2 & 2300 \\
\hline
\end{tabular}

According to the Zimmermann method described by [17], the displacement of the rail due to a quasistatic load can be expressed as

$$
\frac{d^{4} z}{d x^{4}}+\frac{k}{E I} z=0
$$

Imposing the appropriate boundary conditions (point load, symmetry of the deformed shape of the rail, and disappearance of loading effect with distance) leads to the following expression for the vertical displacement developed by [17]

$$
z=\frac{Q}{2 b C} \sqrt[4]{\frac{b C}{4 E I}} e^{-x / L_{v}}\left(\cos \frac{x}{L_{v}}+\sin \frac{x}{L_{v}}\right),
$$

where $Q$ is the transmitted load, $b$ is the gauge, $C$ is an equivalent ballast coefficient, and $L_{v}$ is an elastic length defined as

$$
L_{v}=\sqrt[4]{\frac{b C}{4 E I}}
$$

Once rail vertical displacements have been calculated, by renaming $x=V \cdot t$, where $V$ is the tram speed and $t$ is time, they are differentiated twice with respect to time to obtain the desired accelerations.

2.4. Analytical Solution. Following [16], in order to solve the high complexity that often implies integration of movement equations, the Lamé potentials and Fourier Transform will be used. Thus, the movement of the beam representing the rail can be written as

$$
\begin{aligned}
& \left(A_{K} G k^{2}\left(1+\frac{A_{K} G}{I \rho \omega^{2}-A_{K} G-E I k^{2}}\right)-\rho A \omega^{2}\right) \widetilde{\widetilde{w}}(k, \omega) \\
& =\widetilde{\widetilde{q}}(k, \omega)-a \widetilde{\widetilde{\sigma}}_{Z Z_{1}}(k, 0, \omega) .
\end{aligned}
$$

And the expressions for layers motion expressed in terms of Lamé potentials are

$$
\begin{aligned}
& \frac{d^{2} \tilde{\widetilde{\varphi}}}{d z^{2}}-R_{L}^{2} \widetilde{\widetilde{\varphi}}=0, \\
& \frac{d^{2} \widetilde{\tilde{\psi}}}{d z^{2}}-R_{T}^{2} \widetilde{\widetilde{\psi}}=0,
\end{aligned}
$$

where $\tilde{\widetilde{\varphi}}$ and $\tilde{\widetilde{\psi}}$ are the Lamé potentials expressed in the frequency-wave number domain. The $R_{L}^{2}$ and $R_{T}{ }^{2}$ functions 
represent the longitudinal and shear wave transmission speed, respectively, with their expressions being as follows:

$$
\begin{gathered}
R_{L}^{2}=k^{2}-\frac{\omega^{2}}{c_{L}^{2}-i \omega\left(\lambda^{*}+2 \mu^{*}\right) / \rho}, \\
R_{T}^{2}=k^{2}-\frac{\omega^{2}}{c_{T}^{2}-i \omega \mu^{*} / \rho},
\end{gathered}
$$

where $\lambda^{*}$ and $\mu^{*}$ are the parameters which define the damping of the track and $c_{L}$ and $c_{T}$ represent the velocities of the longitudinal and shear waves in the layer.

Then, the expressions for the displacements (9) and (10) and stresses (11) and (12) can be written as

$$
\begin{aligned}
& \widetilde{\widetilde{u}}(k, z, \omega)=i k \widetilde{\widetilde{\varphi}}-\frac{d \tilde{\widetilde{\psi}}}{d z}, \\
& \widetilde{\widetilde{v}}(k, z, \omega)=\frac{d \widetilde{\widetilde{\varphi}}}{d z}+i k \widetilde{\widetilde{\psi}}, \\
& \widetilde{\widetilde{\sigma}}_{z z}(k, z, \omega)=\tilde{\widehat{\lambda}}\left(\frac{d^{2} \widetilde{\widetilde{\varphi}}}{d z^{2}}-k^{2} \widetilde{\widetilde{\varphi}}\right)+2 \widetilde{\widehat{\mu}}\left(\frac{d^{2} \widetilde{\widetilde{\varphi}}}{d z^{2}}-i k \frac{d \tilde{\widetilde{\psi}}}{d z}\right), \\
& \widetilde{\widetilde{\sigma}}_{z x}(k, z, \omega)=\widetilde{\widetilde{\mu}}\left(2 i k \frac{d \widetilde{\widetilde{\varphi}}}{d z}+\frac{d^{2} \widetilde{\widetilde{\psi}}}{d z^{2}}+k^{2} \widetilde{\widetilde{\psi}}\right) .
\end{aligned}
$$

Solving the system (7) and replacing $\widetilde{\widetilde{\varphi}}$ and $\widetilde{\widetilde{\psi}}$ in (9)-(12):

$$
\begin{aligned}
\widetilde{\tilde{u}}_{j}(k, z, \omega)= & i k\left(A_{1_{j}}(k, \omega) e^{R_{L_{j}} z}+A_{2_{j}}(k, \omega) e^{-R_{L_{j}} z}\right) \\
& +R_{T_{j}}\left(A_{3_{j}}(k, \omega) e^{R_{T_{j}} z}-A_{4_{j}}(k, \omega) e^{-R_{T_{j}} z}\right) \\
\widetilde{\widetilde{v}}_{j}(k, z, \omega)= & R_{L_{j}}\left(A_{1_{j}}(k, \omega) e^{R_{L_{j}} z}-A_{2_{j}}(k, \omega) e^{-R_{L_{j}} z}\right) \\
& -i k\left(A_{3_{j}}(k, \omega) e^{R_{T_{j}} z}+A_{4_{j}}(k, \omega) e^{-R_{T_{j}} z}\right) \\
\widetilde{\sigma}_{Z Z_{j}}(k, z, \omega)= & C_{1_{j}}\left(A_{1_{j}}(k, \omega) e^{R_{L_{j}} z}+A_{2_{j}}(k, \omega) e^{-R_{L_{j}} z}\right) \\
& +C_{2_{j}}\left(A_{3_{j}}(k, \omega) e^{R_{T_{j}} z}-A_{4_{j}}(k, \omega) e^{-R_{T_{j}} z}\right) \\
\widetilde{\widetilde{\sigma}}_{Z x_{j}}(k, z, \omega)= & D_{1_{j}}\left(A_{1_{j}}(k, \omega) e^{R_{L_{j}} z}-A_{2_{j}}(k, \omega) e^{-R_{L_{j}} z}\right) \\
& +D_{2_{j}}\left(A_{3_{j}}(k, \omega) e^{R_{T_{j}} z}+A_{4_{j}}(k, \omega) e^{-R_{T_{j}} z}\right),
\end{aligned}
$$

where

$$
\begin{gathered}
C_{1_{j}}=\left(\widetilde{\widehat{\lambda}}_{j}+2 \widetilde{\widehat{\mu}}_{j}\right) R_{L_{j}}^{2}-\widetilde{\widehat{\lambda}}_{j} k, \\
C_{2_{j}}=-2 i k \widetilde{\widehat{\mu}}_{j} R_{T_{j}}, \\
D_{1_{j}}=2 i k \widetilde{\widehat{\mu}}_{j} R_{L_{j}}, \\
D_{2_{j}}=\widetilde{\widehat{\mu}}_{j}\left(k^{2}+R_{T_{j}}^{2}\right) .
\end{gathered}
$$

Boundary conditions are set so that the displacements between adjacent layers are equal and stresses between them are balanced. Furthermore, no horizontal rail movement and the vanishing of radiated vibrations as the soil depth increases are assumed.

Therefore, replacing the boundary condition of each layer in (13) follows the expression $\mathbf{M} \cdot \mathbf{A}=\mathbf{q}$, where $\mathbf{M}$ is a coefficient matrix, $\mathbf{A}$ is the vector of coefficients to be calculated, and $\mathbf{q}$ is the vector of loads acting on the system.

Following [15], once the motion and stresses expressions have been obtained in terms of deepness and in frequencywave number domain, they are returned to the space-time domain by using the inverse Fourier transform. In order to optimize this process the displacements are written in terms of the wave number domain and then the expression of the inverse Fourier transform is converted into an addition following [14].

The accelerations in each layer are obtained by differentiating twice, with respect to time, the displacement expressions from the inverse Fourier transform.

Total accelerations generated and transmitted to the surrounding ground are obtained through superposition of quasistatic and dynamic overloads.

In order to provide reliability to the model, a measurement camping was carried out as aforementioned. Data, obtained by triaxial accelerometers FastTracer Sequoia, have been processed and compared with those calculated in the model. After calibrating the damping parameters of the layers, the result of comparison between model and real data is shown in Figure 3.

Figure 3 shows the ability of the model to represent the peaks of vibrations and clearly reproduce the effect of passing passenger cars and their bogies through the stretch of the track. However, as shown in Figure 3, measured data (green) is much noisier than the modeled one (red). These noisier accelerogram recordings may be due to the numerous factors intervening in the real vibratory phenomenon such as rails and wheels imperfections. In contrast, only few harmonic loads have been considered in the model to simulate these irregularities. Amplitudes and angular frequencies of these harmonic loads have been obtained after analyzing the Fourier spectrum of the registered data, taking their more significant values. To more accurate results, a larger number of harmonics might be considered, with the drawback of an increased computational time.

It must be highlighted that, due to the typology of track studied, it was not possible to set the accelerometers in the rail, so they were placed next to the elastomeric material where the rail was housed. Then, the data registered with the accelerometers were influenced by the presence of this elastomeric material.

\section{Analysis of Corrugation}

Simulation and analysis of the effect of corrugation in the model presented in the previous section will be addressed. The procedure will be as follows: dynamic overloads caused by the presence of corrugation on the rail will be calculated by using an auxiliary quarter car model and then the influence 


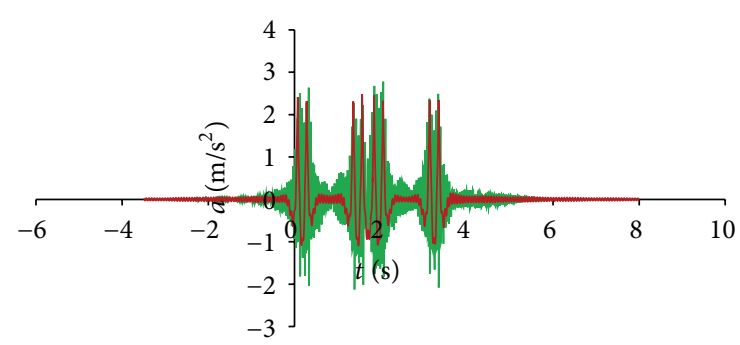

FIGURe 3

in dynamic overloads of parameters which define rail irregularities will be discussed.

Dynamic overloads generate an increase of vibrations and wear, with the first one being the objective of this research. Thus, overloads will feed the model described in Section 2 as harmonic forces in order to obtain the accelerations generated by rail irregularities.

3.1. Quarter Car Model. The vehicle can be modeled in different ways depending on the number of degrees of freedom given to the system: it can range from hundreds of degrees of freedom allowed by commercial multibody systems (MBS) to systems with a single degree of freedom constituted by a mass attached to the track by a spring.

Between both extreme cases, the following can be found: (i) the quarter car model, which will be presented below; (ii) the half car system, it takes into account the sprung masses, the semisprung masses, and the unsprung masses; and (iii) the full car models, these allow us to take into account not only the displacements of masses but also their rotation with respect to each axis.

Nevertheless, from the point of view of the main model and the fact that the loads introduced only have a vertical component, using a full bogie model would be an unnecessary computational expense. Moreover, [18] experimentally demonstrated that the influence on the dynamic stresses of the sprung and semisprung masses is negligible compared to the unsprung masses.

Therefore, to calculate the dynamic overloads generated by corrugation, a quarter car system will be implemented using MATLAB software. In this model, the masses of the vehicle are discomposed in sprung and unsprung masses. The compatibility of strengths and displacements between both masses are solved by a spring and a damping element. Meanwhile, the strength transmission between the unsprung masses and the rail is performed by an equivalent spring, which takes into account not only the stiffness of the railwheel Hertzian contact but also the stiffness of the underlying track.

The expressions governing the behavior of the quarter car model are as follows:

$$
\begin{gathered}
m_{2} x_{2}^{\prime \prime}+k_{2}\left(x_{2}-x_{1}\right)+c_{2}\left(x_{2}^{\prime}-x_{1}^{\prime}\right)=0, \\
m_{1} x_{1}^{\prime \prime}-c_{2} x_{2}^{\prime}+c_{2} x_{1}^{\prime}-k_{2} x_{2}+\left(k_{2}+k_{1}\right) x_{1}-k_{1} z=0,
\end{gathered}
$$

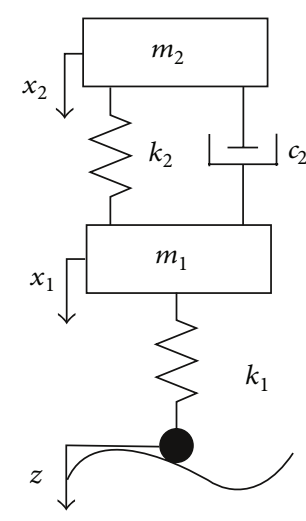

Figure 4

TABLE 2

\begin{tabular}{lc}
\hline \multicolumn{3}{c}{ Quarter car model values } \\
\hline$m_{1}(\mathrm{~kg})$ & 500 \\
$k_{1}(\mathrm{~N} / \mathrm{m})$ & $32,000,000$ \\
$m_{2}(\mathrm{~kg})$ & 4,000 \\
$k_{2}(\mathrm{~N} / \mathrm{m})$ & 501,745 \\
$c_{2}(\mathrm{~N} \cdot \mathrm{s} / \mathrm{m})$ & 875 \\
\hline
\end{tabular}

where: $m_{1}$ : unsprung mass per wheel, $k_{1}$ : contact stiffness, $m_{2}$ : sprung mass per wheel, $k_{2}$ : primary stiffness, $c_{2}$ : primary damping, $x_{i}$ : displacement of the $i$ th mass, $x_{i}^{\prime}$ : velocity of the $i$ th mass, and $x_{i}^{\prime \prime}$ : acceleration of the $i$ th mass.

In Figure 4, a quarter car model sketch can be seen.

In Table 2 the values for the mass, stiffness, and damping are shown.

Problem resolution is performed by the Laplace transference function and, once the accelerations of both masses have been obtained, dynamic overloads are calculated as follows:

$$
F_{\mathrm{dyn}}=m_{1} x_{1}^{\prime \prime}+m_{2} x_{2}^{\prime \prime}
$$

3.2. Sensitivity Analysis. Real rail profiles may be compared to a sum of sinusoids of different amplitudes and wavelengths. Therefore, in order to analyze the effect of a particular corrugation phenomenon, the profile of the affected track is modeled as a sinusoidal function, which is defined by wear amplitude and wavelength. Furthermore, the speed of the train in service is needed to be taken into account due to the dynamic performance of the analysis. When studying the influence of these parameters on the dynamic overloads, an amplitude $A=0.25 \mathrm{~mm}$, a wavelength $\lambda=0.5 \mathrm{~m}$, and a speed $V=50 \mathrm{~km} / \mathrm{h}$ are set. Then, with two of these parameters being fixed, the third one will be modified in order to analyze its influence on dynamic overloads.

It must be highlighted that overload values given for each amplitude, speed, and wavelength correspond to the maximum absolute value of the stationary part of the overload function. The reason for this choice is to consider the most unfavorable case from the point of view of track design, maintenance, and security. 


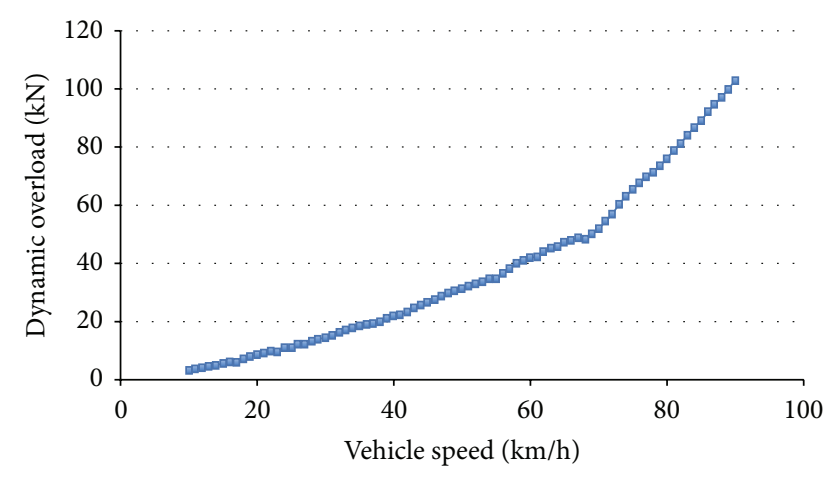

FIGURE 5

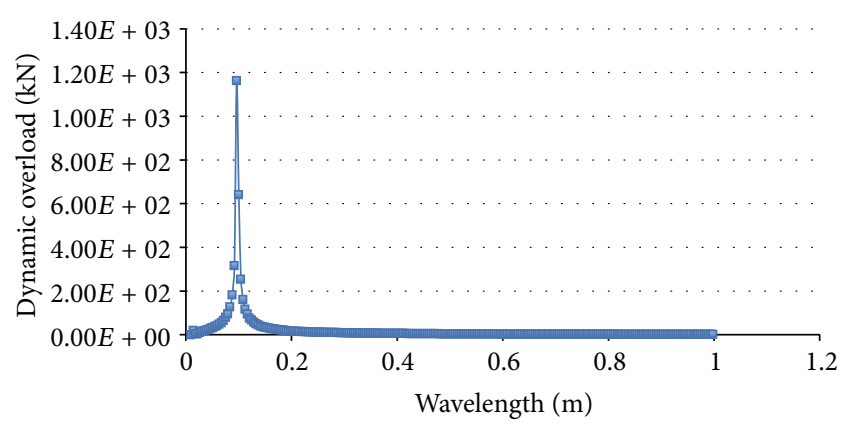

Figure 6

Figure 5 shows the influence of the speed of the tram on dynamic overloads, being the amplitude and wavelength of corrugation: $A=0.25 \mathrm{~mm}$ and $\lambda=0.5 \mathrm{~m}$. From this analysis, it can be affirmed that dynamic overloads grow when increasing the speed of the vehicle.

On the other hand, the influence of wavelength on dynamic overloads, being $A=0.25 \mathrm{~mm}$ and $V=50 \mathrm{~km} / \mathrm{h}$, is shown in Figure 6. In this graph, the peak obtained when the wavelength is about $0.1 \mathrm{~m}$ must be highlighted. Following [19], natural frequencies of the system are calculated as the root of the eigenvalues of

$$
\left|\begin{array}{cc}
\frac{k_{1}+k_{2}}{m_{1}} & \frac{-k_{2}}{m_{1}} \\
\frac{-k_{2}}{m_{2}} & \frac{k_{2}}{m_{2}}
\end{array}\right| .
$$

Then, the natural frequencies are $894.9302 \mathrm{rad} / \mathrm{s}$ and $9.4815 \mathrm{rad} / \mathrm{s}$, the wavelength being associated with first natural frequency: $\lambda=0.0975 \mathrm{~m}$, which corresponds to the wavelength estimated from Figure 6 . Then, it can be said that for this wavelength-being the speed of the vehicle and train and track features constants-a resonance phenomenon will appear.

From Figure 7, it can be noticed that, far from those wavelengths affected by the resonance phenomenon, dynamic overloads decrease when the wavelength of the irregularity increases. That is, the farther the distance between two consecutive irregularities, the lower the forces transmitted to the track.

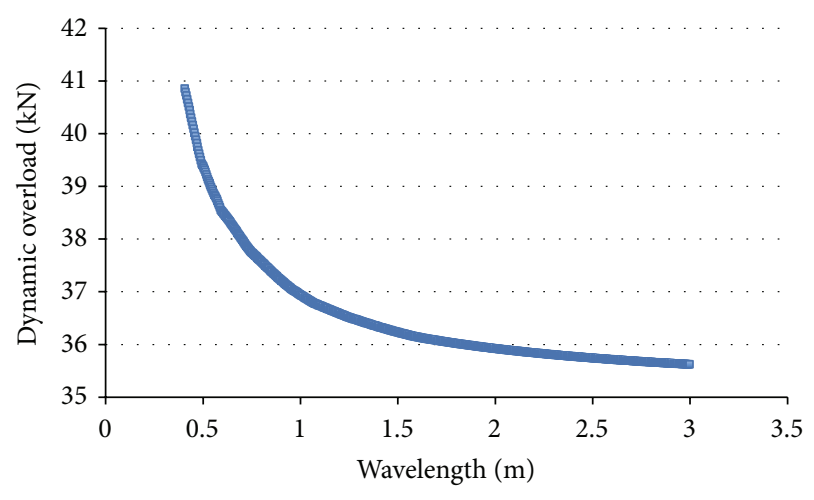

Figure 7

Figure 8 shows the influence of wear amplitude on dynamic overloads, being $\lambda=0.5 \mathrm{~m}$ and $V=50 \mathrm{~km} / \mathrm{h}$. This leads us to affirm that the higher the amplitude, the greater the overload. Furthermore, it must be noticed that overloads and corrugation amplitude are linearly related.

Taking into account the strong nonlinear behavior of the vehicle-track system, the linear behavior of the amplitude of corrugation must be explained. In order to assess the validity of the results obtained with the quarter car model, a multibody model has been implemented with the VAMPIRE commercial software and the influence of the amplitude has been studied. The results are exposed in Figure 9 .

From Figure 9, two different behaviours of the relation amplitude-dynamic overloads are shown. On one hand, when the amplitude of corrugation is lower than $1 \mathrm{~mm}$, the overloads generated by this pathology show a quasilinear behaviour for both models. On the other hand, when the amplitude exceeds this value a nonlinear behaviour can be appreciated for the multibody model but its behaviour remains quasilinear when dynamic overloads are calculated with the quarter car model.

Thus, it can be concluded that the quarter car model described in this paper only is able to be used when the amplitude of the defect considered is lower than $1 \mathrm{~mm}$.

In conclusion, since it is not feasible that amplitudes higher than $1 \mathrm{~mm}$ appear in a tram track as the one proposed in this study and the quarter car model presents the advantage of its simplicity when comparing with the multibody model, the use of this model has been justified. Nevertheless, before using this model for other types of railroads where higher amplitudes may be reached a preliminary study must be carried out.

3.3. Definition of Different Scenarios. Below, a simulation of different scenarios will be performed. Irregularities simulated by the model will be added to the imperfections already present in the calibrated track. The values used in these simulations are shown in Table 3.

Note that in the model a punctual contact between wheels and rails is assumed. Therefore, when simulating wear of a very short wavelength and high amplitude it would be necessary to check the veracity of this hypothesis. Thus, it is 


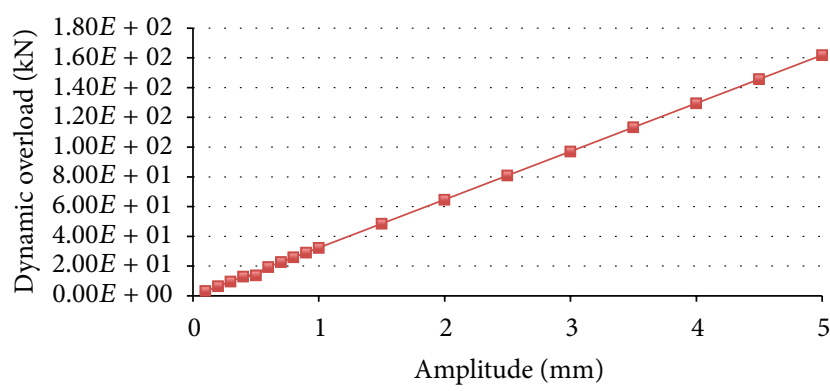

Figure 8

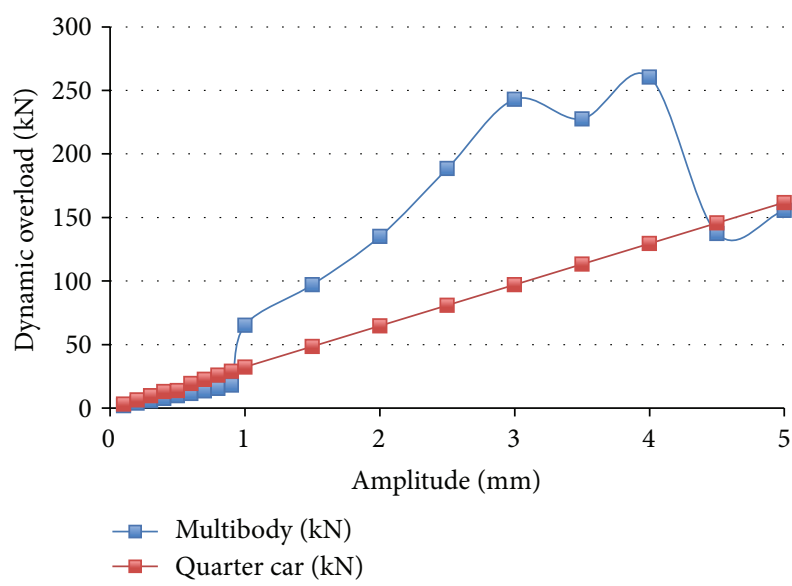

Figure 9

TABLE 3

\begin{tabular}{lccc}
\hline$V(\mathrm{~km} / \mathrm{h})$ & 35 & $\mathbf{5 0}$ & 80 \\
$A(\mathrm{~mm})$ & 0.1 & $\mathbf{0 . 2 5}$ & 0.5 \\
$\lambda(\mathrm{m})$ & 0.3 & $\mathbf{0 . 5}$ & 1 \\
\hline
\end{tabular}

established that the curvature of the sinusoid representing the rail must be greater than wheel curvature.

Wheel curvature is $1 / R_{w}$.

The curvature of the sinusoid representing the rail can be expressed as $y=-A \sin (k x)$ so: $y=-A \cdot \sin (2 \pi x / \lambda)$. By differentiating twice with respect to the position:

$$
y^{\prime \prime}=\frac{4 \pi^{2}}{\lambda^{2}} \cdot A \cdot \sin \left(\frac{2 \pi x}{\lambda}\right) .
$$

So the maximum curvature of the rail is $\left(4 \pi^{2} / \lambda^{2}\right) A \mathrm{~m}^{-1}$.

Relating both curvatures,

$$
\frac{1}{R_{w}} \leq \frac{4 \pi^{2}}{\lambda^{2}} A
$$

Then, the wavelength of the irregularity considered must satisfy

$$
\lambda \geq 2 \pi \sqrt{A R_{w}} .
$$

After verifying that all of them satisfy (20), the scenarios are discussed in Table 4.
TABLE 4

\begin{tabular}{lccccc}
\hline Scenario & $\begin{array}{c}V \\
(\mathrm{~km} / \mathrm{h})\end{array}$ & $\begin{array}{c}A \\
(\mathrm{~mm})\end{array}$ & $\begin{array}{c}\lambda \\
(\mathrm{m})\end{array}$ & $F_{\text {din max }}(\mathrm{N})$ & $\begin{array}{c}a_{\max } \\
\left(\mathrm{m} / \mathrm{s}^{2}\right)\end{array}$ \\
\hline Case 1 & 35 & 0.25 & 0.5 & $1.7783 e+04$ & 2.44511 \\
Case 2 & 50 & 0.25 & 0.5 & $3.8228 e+04$ & 4.77442 \\
Case 3 & 80 & 0.25 & 0.5 & $1.0621 e+05$ & 12.3465 \\
Case 4 & 50 & 0.1 & 0.5 & $1.5291 e+04$ & 4.62642 \\
Case 5 & 50 & 0.5 & 0.5 & $7.6456 e+04$ & 4.92609 \\
Case 6 & 50 & 0.25 & 0.3 & $1.1547 e+05$ & 5.69606 \\
Case 7 & 50 & 0.25 & 1 & $8.4703 e+03$ & 4.01358 \\
\hline
\end{tabular}

From the results for the dynamic overloads, the behavior predicted by earlier Figures $5-8$ is confirmed: high speed, high wavelengths, and low amplitudes generate higher values for dynamic overloads.

Furthermore, in general, the higher the dynamic overload generated by corrugation, the greater the maximum accelerations generated and transmitted to the track. However, this affirmation is not satisfied in all cases (see cases 1 and 7). As discussed in Section 2, this is due to the fact that the whole behavior of the system is determined by not only dynamic overloads, but also the quasistatic ones, which depend on speed. Thus, although in case 7 lower dynamic overloads have been obtained compared to case 1, the influence of the vehicle speed causes the greater accelerations generated in case 7.

\section{Conclusions}

The aim of this paper was to implement a model able to reproduce the vibratory behavior of a tram railroad, where a measurement campaign was conducted. Then, after confirming its correct behavior, a simulation of corrugation in that track was performed.

For this purpose, an analytical model has been implemented in the Mathematica software based on that developed by [16]. Subsequently, a morphological calibration was carried out.

An auxiliary quarter car model was implemented to evaluate the dynamic overloads generated by corrugation, and the following conclusions were obtained: the greater the speed of the vehicle and the amplitude of the defect, the greater the dynamic overloads transmitted to the track. On the other hand, the higher corrugation wavelength, the lower overloads generated.

Dynamic overloads obtained as stated before feed the main model to obtain the vibrations generated by rail corrugation. From this analysis, it may be concluded that the overloads transmitted to the track due to corrugation are highly influential in the maximum acceleration generated by the vehicle, but also the effect of vehicle speed is very significant.

The model described in this paper may be used as an aid to maintenance of railway infrastructure. Overall, for a given stretch of track, tram vehicles usually travel at the same speed. Moreover, according to [2] given the main features of a stretch of track and of those vehicles traveling through 
it, wavelength of rail corrugation may be predicted. Then, being the maximum allowable values for vibrations fixed for a particular stretch of track, model can predict from which corrugation amplitude maintenance operations are needed.

\section{Conflict of Interests}

The authors declare that there is no conflict of interests regarding the publication of this paper. The aim of this paper is to publish the results of the research conducted considering only academic purposes.

\section{References}

[1] D. Thomson, Railway Noise and Vibration. Mechanisms, Modeling and Means of Control, Elsevier, 2009.

[2] S. L. Grassie and J. Kalousek, "Rail corrugation: characteristics, causes and treatments," Journal of Rail and Rapid Transit, vol. 207, no. 1, pp. 57-68, 1993.

[3] S. L. Grassie, "Rail corrugation: advances in measurement, understanding and treatment," Wear, vol. 258, no. 7-8, pp. 12241234, 2005.

[4] O. Oyarzábal, N. Correa, E. G. Vadillo, J. Santamaría, and J. Gómez, "Modelling rail corrugation with specific-track parameters focusing on ballasted track and slab track," International Journal of Vehicle Dynamics and Mobility, vol. 49, no. 11, pp. 1733-1748, 2011.

[5] N. Correa, O. Oyarzábal, E. G. Vadillo, J. Santamaría, and J. Gómez, "Rail corrugation development in high speed lines," Wear, vol. 271, no. 9-10, pp. 2438-2447, 2011.

[6] J. I. Egaña, J. Vinolas, and M. Seco, "Investigation of the influence of rail pad stiffness on rail corrugation on a transit system," Wear, vol. 261, no. 2, pp. 216-224, 2006.

[7] E. Tassilly and N. Vincent, "A linear model for the corrugation of rails," Wear, vol. 150, no. 1, pp. 25-45, 1991.

[8] R. Ferrara, G. Leonardi, and F. Jourdan, "Numerical modelling of train induced vibrations," in Proceedings of the 5th International Congress on Sustainability of Road Infrastructures, p. 155, Elsevier, Rome, Italy, 2012.

[9] X. S. Jin, Z. F. Wen, K. Y. Wang, Z. R. Zhou, Q. Y. Liu, and C. $\mathrm{H}$. Li, "Three-dimensional train-track model for study of rail corrugation," Journal of Sound and Vibration, vol. 293, no. 3-5, pp. 830-855, 2006.

[10] X. Lei and J. Wang, "Dynamic analysis of the train and slab track coupling system with finite elements in a moving frame of reference," Journal of Vibration and Control, pp. 1-17, 2013.

[11] J. I. Real, T. Asensio, L. Montalbán, and C. Zamorano, "Analysis of vibrations in a modeled ballasted track using measured rail defects," Journal of Vibroengineering, vol. 14, no. 2, pp. 880-893, 2012.

[12] M. Melis, Introducción a la Dinámica Vertical de la vía y Señales Digitales en Ferrocarriles, UPM, Madrid, Spain, 2008.

[13] J. Real, P. Martínez, L. Montalbán, and A. Villanueva, "Modelling vibrations caused by tram movement on slab track line," Mathematical and Computer Modelling, vol. 54, no. 1-2, pp. 280291, 2011.

[14] P. Salvador, J. Real, C. Zamorano, and A. Villanueva, "A procedure for the evaluation of vibrations induced by the passing of a train and its application to real railway traffic," Mathematical and Computer Modelling, vol. 53, no. 1-2, pp. 42-54, 2011.
[15] A. V. Metrikine and A. C. W. M. Vrouwenvelder, "Surface ground vibration due to a moving train in a tunnel: twodimensional model," Journal of Sound and Vibration, vol. 234, no. 1, pp. 43-66, 2000.

[16] P. M. Koziol, C. Mares, and I. Esat, "Wavelet approach to vibratory analysis of surface due to a load moving in the layer," International Journal of Solids and Structures, vol. 45, no. 7-8, pp. 2140-2159, 2008.

[17] A. López Pita, Infraestructuras ferroviarias, Edicions UPC, Barcelona, Spain, 2006.

[18] A. Proud'Homme, "La Voie," Revue Générale des Chemins de Fer, no. 1, pp. 56-72, 1970.

[19] S. Timoshenko and D. Young, Vibration Problems in Engineering, D. Van Nostrand, New York, NY, USA, 1961. 


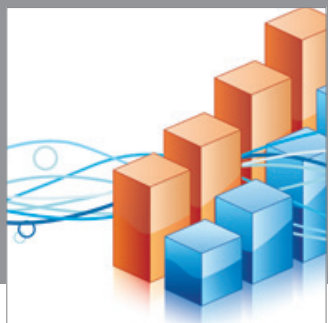

Advances in

Operations Research

mansans

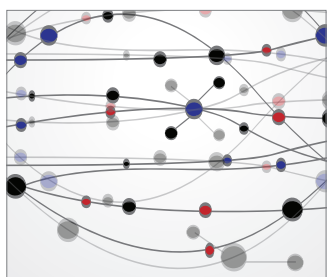

The Scientific World Journal
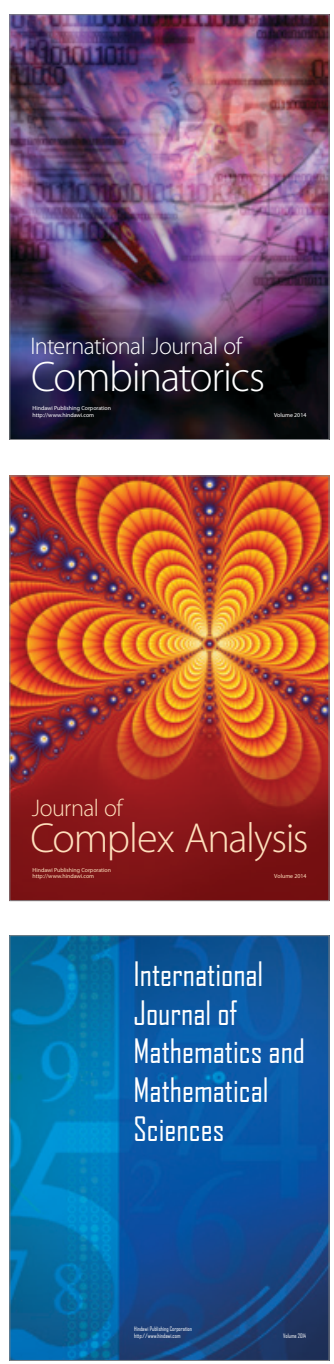
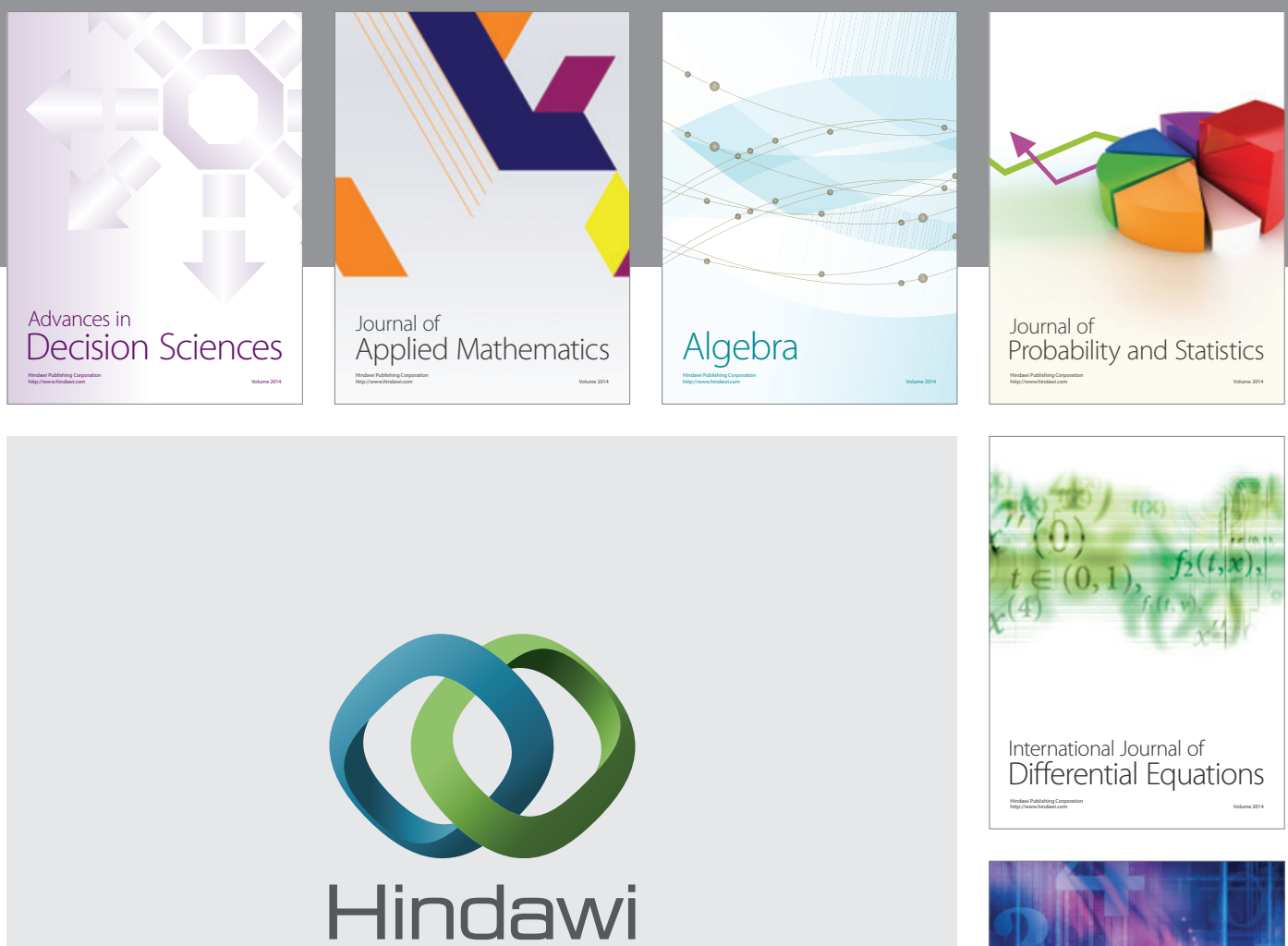

Submit your manuscripts at http://www.hindawi.com
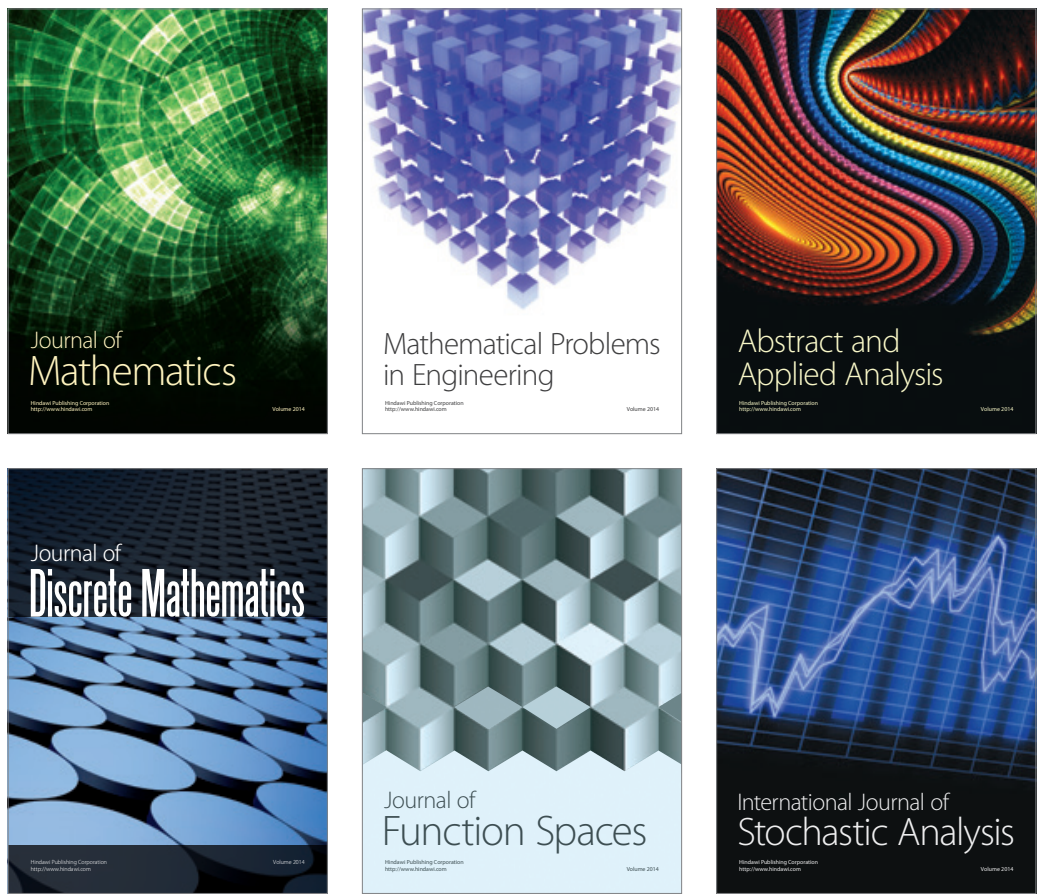

Journal of

Function Spaces

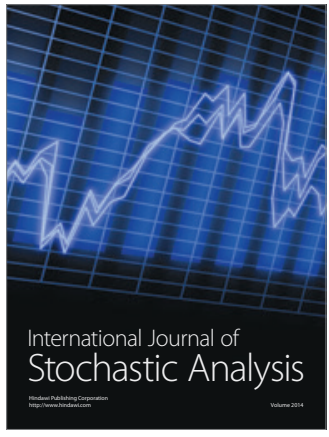

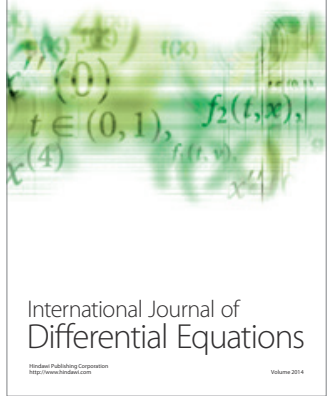
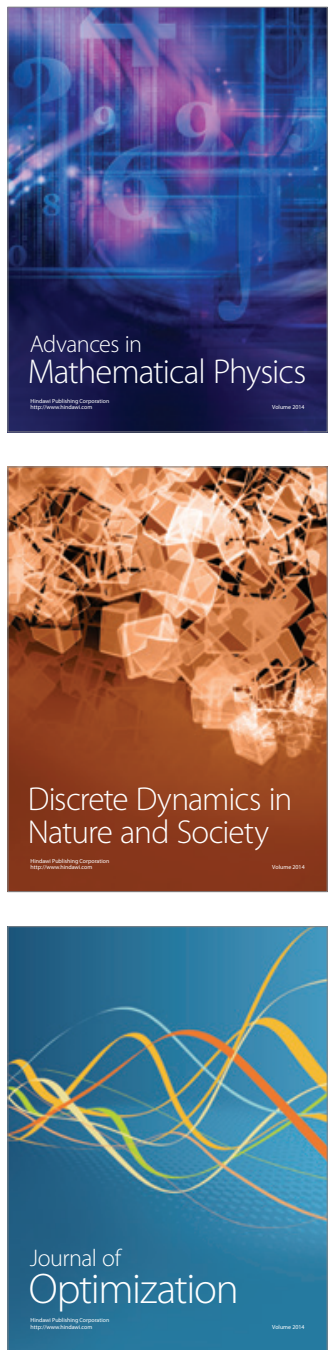